DOI: 10.4274/jarem.galenos.2018.2527

J Acad Res Med 2020; 10(1): 100-2

\title{
Treatment of Sympathetic Nerve Neurofibroma of the Neck and Approach to the Postoperative Pain
}

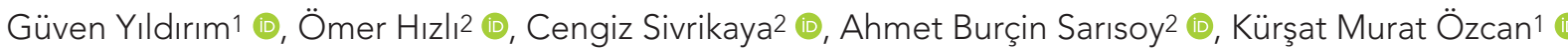 \\ ${ }^{1}$ Giresun University Faculty of Medicine, Department of Otolaryngology, Giresun, Turkey \\ ${ }^{2}$ Giresun University, Prof Dr. A. Illhan Özdemir Training and Research Hospital, Clinic of Otolaryngology, Giresun, Turkey
}

Cite this article as: Yıldırım G, Hızı Ö, Sivrikaya C, Burçin Sarısoy A, Özcan KM. Treatment of Sympathetic Nerve Neurofibroma of the Neck and Approach to the Postoperative Pain. JAREM 2020;10(1): 100-2

\begin{abstract}
Peripheral nerve sheath tumors (PNST) are a kind of neuro-ectodermal tumors. Neurofibromas are benign PNST originating from Schwann cells, fibroblast-like cells, and perineural cells. The origin of symptomatic neurofibromas may be many parts of the body, but about $25 \%$ of them originate from the head and neck region. Postoperative pain is a quite common condition in these tumors of which primary treatment is surgical excision. Postoperative treatment of this neuropathic pain which is unresponsive to many painkillers is still an important problem. In this case study, we discussed our applications and results of a 64-year-old female patient with sympathetic nerve neurofibroma to relieve the postoperative pain.

Keywords: Sympathetic nerve, neurofibroma, postoperative pain
\end{abstract}

\section{INTRODUCTION}

Peripheral nerve sheath tumors (PNST) are tumors of neuroectodermal origin (1). Neurofibromas are benign PNST originating from Schwann cells, fibroblast-like cells and perineural cells (2). Neurofibromas can originate from any peripheral nerve. Symptomatic neurofibromas can be seen in many parts of the body, but they can be seen in the head and neck region at the rate of about $25 \%$. (3). These tumors are usually asymptomatic until they reach the size that will cause discomfort or deformity. Approximately half of neurofibromas are manifested by pain (4). The characteristic physical examination finding of PNST is a mass that cannot be manipulated up and down but moves only laterally. Although the majority of neurofibromas in the head and neck are benign, slowly growing tumors, it has been reported that malignant degeneration may also occur (5).
The purpose of the treatment of these tumors is to provide resection by maintaining the function of the nerve. Intracapsular enucleation of PNST is defined as a surgical technique that can limit the risk of nerve damage without increasing the risk of tumor recurrence (6). However, post-surgical pain is a common condition in these tumors. Elimination of this neuropathic pain that remains unresponsive to many painkillers continues to be an important problem. In this study, we discussed the practices performed to relieve pain after surgery and their results in a 64-year-old female patient with sympathetic nerve neurofibroma.

\section{CASE PRESENTATION}

Our patient was a 64-year-old woman who first noticed a mass in her neck 3 months ago. The patient applied to our clinic because she felt that the mass was growing constantly and at

ORCID IDs of the authors: G.Y. 0000-0003-3864-3522; Ö.H. 0000-0001-6822-2679; C.S. 0000-0001-8670-5156; A.B.S. 0000-0002-2447-007X

K.M.Ö. 0000-0002-5262-0565. 
admission, she had no pain or sensitivity. He did not show the symptoms of dysphagia, dysphonia, hearing changes, cranial nerve, and brachial plexus. On physical examination, it was observed that there was a hard mass that was localized in the left anterior cervical region and that was mobile with palpation. In magnetic resonance imaging (MRI), a mass deeply well confined with the left sternocleidomastoid muscle (SCM), hyperintense in T2 sequences, and isointense in T1 sequences was detected (Figure 1). The mass showed heterogeneous enhancement and its dimensions were measured as $4.0 \times 4.4 \times 6.2 \mathrm{~cm}$. Diffusion imaging showed a mass with an apparent diffusion coefficient of $2 \times 10-3$ $\mathrm{mm} 2 / \mathrm{sec}$. Imaging findings were consistent with a neurofibroma. Upon the decision of surgical treatment, informed consent was obtained from the patient after giving detailed information about the operation. In addition, she was informed about that a case presentation regarding all diagnosis-treatment stages that would be applied to the patient and their results would be prepared and presented to the literature, and her approval was obtained.

The patient was operated under general anesthesia. After endotracheal intubation, a skin incision was made at the anterior border of the SCM from top to bottom and the subplatysmal skin flap was elevated to both sides. The mass was revealed by palpating and dissecting from surrounding tissues (Figure 2). The mass lateralized the internal jugular vein (IJV) and was causing compression. Likewise, the vagus nerve and carotid artery lateralized by pushing from below. Dissection was continued along the lower margin of the mass and the XI. cranial nerve was identified; The XII. cranial nerve was on the upper edge of the mass. The mass extended to the prevertebral muscles and the base of the skull over the deep cervical fascia. When the lower part of the tumor was determined and followed, it was observed that the mass separated from the vagus and originated from the sympathetic nerve. Later, IJV and carotid artery were isolated from the mass by blunt dissection. The mass was resected by maintaining the vagus. Histopathological examination was reported as neurofibroma (Figure 3).

The patient who had no postoperative complications was discharged in good condition. However, in the following period, unbearable pain occurred in the patient, which radiated to the neck and increased during eating. The given non-steroidal painkillers did not eliminate pain. The patient was consulted to

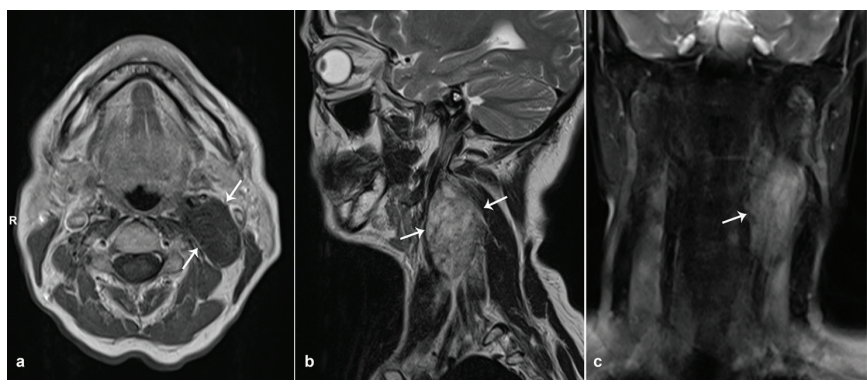

Figure 1. Magnetic resonance imaging. a) Isointense image of the mass in T1 sequence axial section, b) hyperintense view of the mass in T2 sequence sagittal section, c) T2 sequence coronal section the pain outpatient clinic, $25 \mathrm{mg}$ of amitriptyline once a day and $150 \mathrm{mg}$ of pregabalin, one in the morning in the first week and one in the morning and in the evening in the second week, were initiated. Although the pain decreased with these treatments, it did not disappear completely. $4 \mathrm{cc}$ injection consisting of $1 \mathrm{cc}$ prednisolone and $3 \mathrm{cc}$ bupivacaine combination was applied to the resection region of the patient, who insistently stated that she felt pain especially during eating, by interventional radiology. After the injection, the patient's pain decreased, and the pain disappeared over time. The patient, who come for intermittent control, has not had pain for 4 months.

\section{DISCUSSION}

Serious pain after neurofibroma excision is a fairly common condition. In the treatment of postoperative pain, besides medical treatments with a multidisciplinary approach, interventional

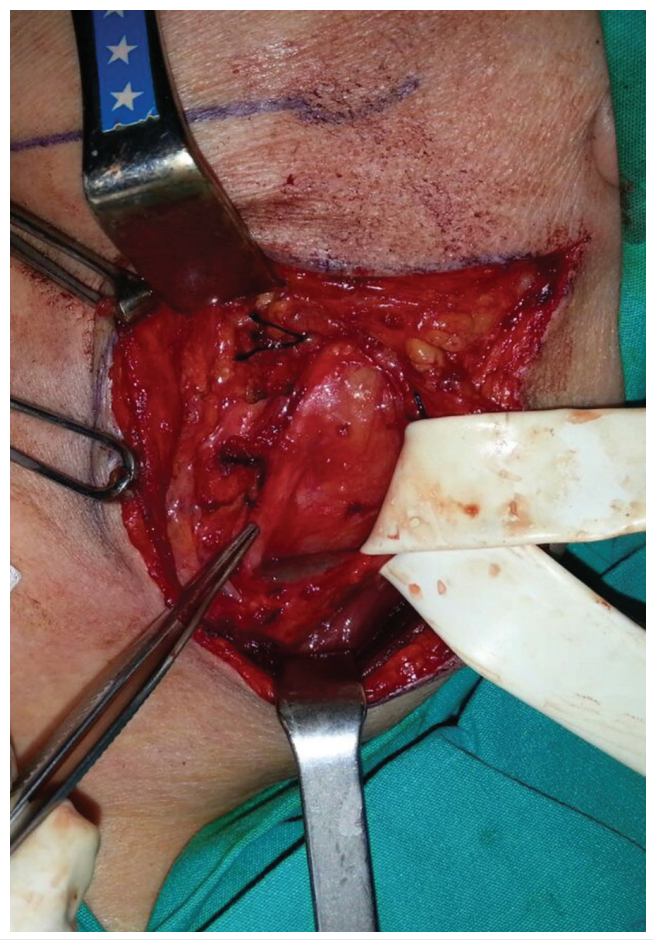

Figure 2. Intraoperative view

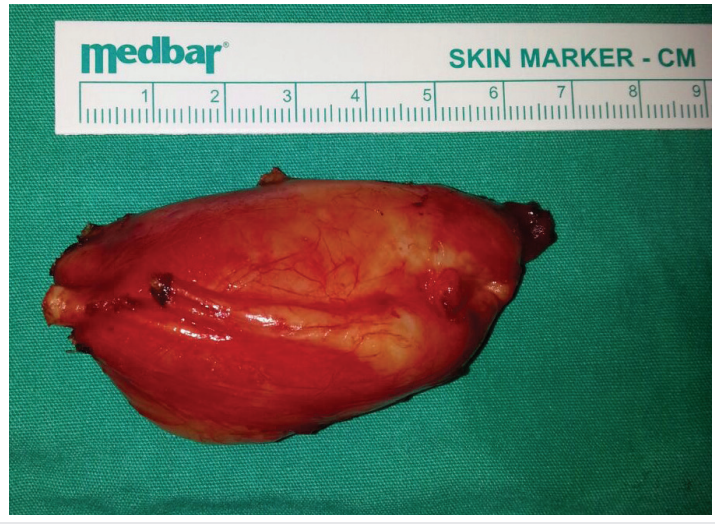

Figure 3. View after excision 
procedures may also be required. If a PNST is suspected with history and physical examination/ultrasonography (USG) findings, it would be reasonable to obtain an MR image of the mass in the next step. MRI is considered as the best imaging method to characterize PNST $(7,8)$. In our case, the location and dimensions of the mass were first determined by USG, and it was revealed that there was a solid mass. Afterwards, MRI was performed to reveal its relationship with surrounding tissues and blood supply status and to rule out a possible carotid body tumor. As shown in this case report, neurofibromas are usually isointense in $\mathrm{T} 1$ sequences and hyperintense in $\mathrm{T} 2$ sequences, and they are observed heterogeneously in $\mathrm{T} 1$ after gadolinium contrast $(7,8)$. Benign and malignant primary nerve sheath tumors exhibit overlapping features such as T2 hyperintensity, focal enlargement and strengthening in MRI. In our case, we determined that the mass might be a tumor of nerve origin with MRI.

Nerve tumors can be either benign or malignant. Malignant tumors are generally larger than benign tumors. While myxoid, cystic and hyaline changes are associated with benign tumors, necrosis, bleeding and mitotic activity were observed in malignant tumors (1). Also, while it is benign first, malignant change can be observed over time. In the literature, a case of solitary-sclerotic neurofibroma showing malignant transformation following three recurrences has been reported (9).

While there is a nerve fiber that enters and exits the tumor in the schwannomas, multiple fibers pass through the tumor mass in the neurofibroma. The basis of neurofibroma treatment is based on the resection of the tumor with the preservation of the function of these nerves, which may be associated with the tumor to varying degrees. Donner et al. (10) recommended intracapsular enucleation of neurofibroma in surgical treatment. They reported that more than $10 \%$ of patients developed pain syndromes related to the sensory area in which the operated nerve spread. In such cases, reaching the mass completely is an important problem. An improper exposition can cause injury to other vital organs. Therefore, in our case, an incision from the mastoid tip to the calvicula in front of the SCM was made to create a large surgical area. This was critical to prevent damage to important neurovascular structures that crossed the mass. Imaging is helpful in diagnosis and can also reveal safe surgical corridors for the surgeon. For safe tumor resection, electromyography, which evaluates nerve action potentials in the intraoperative period, can also be used (2).

The primary goal of both surgical and radiosurgery treatment of nerve tumors in the craniocervical junction is the preservation or restoration of the functions of the underlying cranial nerves. However, after treatment, head and neck pain may occur. In patients with nerve sheath tumors in the neck region, pain can be observed before surgery, and in the occipital region, in the right parietal and temporal areas, there may be pain radiating to the neck and shoulder. In our case, there was a very mild pain that was not disturbing before the operation, while severe pain occurred after the operation. Before, simple painkillers were given considering that as the pain of the surgical site. However, when the pain did not ease, support was obtained from the pain and anesthesia polyclinic. Despite the pregabalin treatment initiated by the pain and anesthesia outpatient clinic, the pain did not disappear completely, and interventional radiology blocked the exposed nerve fibers in the resection area of the patient.

Even if the nerve sheath tumors are benign, complete excision after the operation can sometimes lead to very severe pain. In these cases, firstly, simple painkillers should be tried parenterally or orally, and in cases which gives no response, support should be obtained from the pain outpatient clinic. A nerve blockage application that will be applied by interventional radiology can be considered as a last resort.

Informed Consent: Informed consent was obtained from the patient after giving detailed information about the operation.

Peer-review: Externally peer-reviewed.

Author Contributions: Surgical and Medical Practices - G.Y., A.B.S., K.M.Ö.; Concept - Ö.H., C.S.; Design - C.S.; Analysis and/or Interpretation - Ö.H., C.S., A.B.S.; Literature Search - G.Y., K.M.Ö.; Writing Manuscript G.Y., Ö.H.

Conflict of Interest: The authors have no conflict of interest to declare.

Financial Disclosure: The authors declared that this study has received no financial support.

\section{REFERENCES}

1. Chikkannaiah $P$, Boovalli MM, Nathiyal V, Venkataramappa S. Morphological spectrum of peripheral nerve sheath tumors: An insight into World Health Organization 2013 classification. J Neurosci Rural Pract 2016; 7: 346-54.

2. Bray DP, Chan AK, Chin CT, Jacques L. Large cervical vagus nerve tumor in a patient with neurofibromatosis type 1 treated with gross total resection: Case report and review of the literature. J Brachial Plex Peripher Nerve Inj 2016; 11: e48-e54.

3. Peetermans JF, Van de Heyning PH, Parizel PM, Martin JJ, Forton GE, Vanmaele RG, et al. Neurofibroma of the vagus nerve in the head and neck: A case report. Head Neck 1991; 13: 56-61.

4. Marocchio LS, Oliveira DT, Pereira MC, Soares CT, Fleury RN. Sporadic and multiple neurofibromas in the head and neck region: a retrospective study of 33 years. Clin Oral Investig 2007; 11: 165-9.

5. Scheithauer BW, Erdogan S, Rodriguez FJ, Burger PC, Woodruff JM, Kros $\mathrm{JM}$, et al. Malignant peripheral nerve sheath tumors of cranial nerves and intracranial contents: a clinicopathologic study of 17 cases. Am J Surg Pathol 2009; 33: 325-38.

6. Raguin T, Alhabib SF, Schultz P, Dupret-Bories A. [Surgical treatement by intracapsular enucleation of cervical peripheral neural sheath tumors]. Rev Laryngol Otol Rhinol (Bord) 2015; 136: 45-4.

7. Kuntz Ct 4th, Blake L, Britz G, Filler A, Hayes CE, Goodkin R, et al. Magnetic resonance neurography of peripheral nerve lesions in the lower extremity. Neurosurgery 1996; 39: 750-7.

8. Singh T, Kliot M. Imaging of peripheral nerve tumors. Neurosurg Focus 2007; 22: 1-10

9. Moriya S, Hirose J, Yoshida Y, Yamamoto O. Malignant peripheral nerve sheath tumour arising from solitary sclerotic neurofibroma. Acta Derm Venereol 2016; 96: 706-7.

10. Donner TR, Voorhies RM, Kline DG. Neural sheath tumors of major nerves. J Neurosurg 1994; 81: 362-73. 